\title{
El otro precio de las becas y subvenciones
}

\section{The other price of grants}

Recientemente, se ha generado entre colegas un debate sobre el acceso a congresos. Ello se ha dado tanto a partir de la distribución, por parte de los laboratorios medicinales, de becas para el Congreso del Centenario, como del amable reclamo a la Sociedad Argentina de Pediatría para que otorgue, a todo asociado con su cuota al día, pases libres para sus propios congresos. Dado lo interesante del tema, me atrevo a compartir algunas reflexiones.

$\mathrm{Al}$ recibir becas o subvenciones para congresos tengo la misma sensación de incomodidad que vivo al subirme a un colectivo y leer "este boleto está subsidiado por el Estado Nacional". Lo mismo me ocurre al recibir las facturas de luz y gas, aunque las naturalezas del subsidio y la beca a un congreso sean diferentes. Aclaro que he aceptado becas y que si tuviera un Agente de Propaganda Médica conocido, posiblemente seguiría aceptando alguna. Sin embargo, no dejo de hacerme preguntas, ni de sentir emociones que me llevan a cuestionarme si deseo que me subsidien, si quiero que me bequen. El motivo de estas preguntas es que desconozco el precio final que eso tiene para mí y para la sociedad.

La posesión de cualquier cosa implica derechos y obligaciones. ¿A qué me obliga el obtener una beca que obviamente no pagué ni merecí, al menos por un medio explícito, claro y transparente? No dejo de preguntarme si quiero recibir el fruto del esfuerzo ajeno, si en realidad deseo dejar de pagar por algo que otra persona, aun con intereses profesionales parecidos a los míos, puede estar dispuesta a pagar. Entiendo que el uso que hace alguien de su dinero y de su tiempo es un fiel reflejo de sus valores. La preocupación por la capacitación profesional muestra algo muy positivo; se evidencia en la disposición a invertir nuestro tiempo en ella. Pero... ¿y el dinero? ¿Tenemos la misma disposición para invertirlo en similar medida? Si acepto que otros inviertan dinero por mí, existe una respuesta implícita: no estoy dispuesta a dar mi dinero en la misma medida que mi tiempo. Al menos no para congresos formativos. Y esta salvedad es interesante, porque se puede contemplar a los congresos con, al menos, igual peso social que académico; desde este punto de vista, algunos aceptan gustosos las becas en tanto invitaciones a un evento social y formativo a la vez.

La idea de que una entidad organizadora como la SAP lograra subvencionar a sus asociados para la asistencia a sus congresos, resulta realmente ingenua considerando el moderado monto de nuestras cuotas. Otra posibilidad sería que los patrocinadores le pagaran a la organización, de modo que, para los asistentes, la asistencia fuera gratuita. Ahorraría, es cierto, algunos problemas, pero el tema de fondo seguiría siendo el mismo: ¿es lógico que de un conjunto de trabajadores profesionales, universitarios y con posgrados, un buen número no pueda (y otro no quiera) pagar para asistir a un congreso profesional formativo?

$\mathrm{Mi}$ anhelo es recibir lo que merezco por $\mathrm{mi}$ labor como médica y pagar (sin dudar) de mi propio bolsillo la asistencia a un congreso. ¿Estaré muy desubicada en este mundo por pensar y sentir así? No lo sé. Sí sé que soy un ser humano adulto, responsable, libre, autónomo, capaz de bastarme a mí misma -quizás, en forma desigualen lo material, psicológico y espiritual. Entonces, me pregunto si estos regalos son dignos, si no habrá algo en mí que se deteriore paulatinamente al recibirlos, si no habrá algo que vaya cambiando en mi naturaleza al tolerarlos. Estas dudas ya son parte del precio a pagar. Otros costos potenciales incluyen reparos morales y científicos: ¿me sentiré obligada a corresponder al patrocinador, recetando sus medicamentos? ¿Me veré impedida de recetarlos, justamente por haberme patrocinado? Incluso creyendo que un medicamento del laboratorio en cuestión sea el mejor disponible, ¿podré convencer a mi propio juicio y conciencia de que mi decisión de recetarlo no esté sesgada?

Aspiro a diario a que el apotegma de "...en un mundo mejor" no sea solo una frase, sino una meta a la que intento aproximarme con mis pequeñas decisiones de todos los días. Además, en mi opinión, la transformación del mundo empieza con la del propio mundo: el interno. Esa transformación supone varios precios a pagar: a veces, se trata de hacer cosas diferentes para obtener resultados diferentes. En mi caso, otras veces cedo: no interpuse un amparo legal para intentar que no se subsidiara mi cuenta de luz, por ejemplo, pero no puedo dejar de preguntarme si aceptar que me otorguen una "beca" (pudiendo pagar lo que corresponde), contribuye a un mundo mejor.

Asimismo, tampoco puedo dejar de preguntarme si, al no pagar lo que me corresponde, estoy contribuyendo, en realidad, a un mundo peor.

Dra. Paula Pradines

Centro de Salud y Atención Primaria "La Cunita II"

Municipalidad de Ezeiza pradines.paula@gmail.com

http:/ /dx.doi.org/10.5546/aap.2012.4 


\section{Farmacología en pediatría y usos de medicamentos no autorizados Pediatric pharmacology and use of unapproved drugs (off label)}

Desde el año 1995, la Academia Estadounidense de Pediatría aborda el tema del uso de fármacos en neonatos y niños ${ }^{1}$ fundamentalmente debido a su utilización fuera de lo especificado en el prospecto (off label) o por no estar autorizados para su empleo en niños.

La prescripción farmacológica fuera de prospecto aumenta el margen habitual de incertidumbre, pues se desconocen evaluaciones y mediciones precisas del beneficio por obtener, así como del riesgo asumido. El uso pediátrico de medicamentos off label genera entre los pediatras, particularmente neonatólogos e internistas, el dilema entre privar a un paciente de la medicación posiblemente adecuada, iniciar tratamientos con medicación insuficiente o extrapolar a la población pediátrica la información correspondiente a los adultos. ${ }^{2}$

En nuestro medio, se estima que para las unidades de internación de clínica pediátrica en un hospital de tercer nivel, el número de prescripciones de medicamentos fuera de prospecto rondaría el $40 \%{ }^{3}$ y en las unidades de neonatología el número es mayor.

Es totalmente comprensible el temor a generar daño a través de un ensayo clínico cuando el medicamento ya se utiliza de manera off label, pero se crea la situación paradojal de reemplazar una investigación correctamente diseñada, por otra a escala individual, repetida innumerables veces, sin registro ordenado de los resultados, con baja probabilidad de poder generalizarse y que, como ya se ha demostrado, aumenta el riesgo de errores en la prescripción. ${ }^{4}$

Existen iniciativas locales de ensayos farmacológicos de farmacocinética y farmacodinamia en pediatría, como los desarrollados para el tratamiento del beznidazol en niños con enfermedad de Chagas, ${ }^{5,6}$ que contribuyen a dar respuesta a la problemática no solo desde la terapéutica, sino también desde los aspectos regulatorios de la investigación farmacológica independiente en niños de nuestro medio.

Entre los años 2008 y 2010 se han registrado en Clinical Trials.gov 22 ensayos clínicos pediátricos en la Argentina y otros 10 que incluían niños y adultos, ${ }^{7}$ pero en relación a los estudios que se desarrollan en adultos el número continúa siendo escaso.
Resta mucho camino por recorrer en este campo, no solo a nivel local, sino desde la perspectiva global.

"El tren ya arrancó", fueron las palabras del Dr. Kalle Hopu, Presidente de la Sección de Farmacología Clínica Pediátrica de la International Union of Pharmacology (IUPHAR), en la Primera Reunión Latinoamericana de Farmacología Pediátrica, desarrollada en forma conjunta con la Subcomisión de Investigación de la Sociedad Argentina de Pediatría en octubre de este año.

En ella se destacó la necesidad de incorporar la farmacología pediátrica en los cursos de formación en farmacología general y jerarquizar el problema de los off label, fundamentalmente en neonatología, enfermedades olvidadas y embarazadas.

Esta reunión ha nucleado a la mayoría de los actores locales que desde hace años trabajan para el desarrollo de la farmacología pediátrica en la Argentina y es posible que las acciones conjuntas de la Sociedad Argentina de Pediatría, IUPHAR y ANMAT permita continuar desarrollando conocimiento de vital importancia en la terapéutica pediátrica.

\section{Dr. Norberto Giglio Hospital de Niños Ricardo Gutiérrez Buenos Aires}

http:/ /dx.doi.org/10.5546/aap.2012.5

1. Committee on Drugs. Guidelines for ethical conduct of studies to evaluate drugs in pediatric populations. Pediatrics 1995; 95:286-294

2. Giglio ND, MalozowskiS. Prescripciones fuera de prospecto. Arch Argent Pediatr 2004;102(2):1-4.

3. Giglio N, Rodríguez Gesto E, Arnaldez F, Grichener J. Empleo de medicamentos en un hospital pediátrico: Usos validados y no validados. $33^{\circ}$ Congreso Argentino de Pediatría. Mar del Plata, octubre 2003.Pág.166-SP448.

4. Conroy S. Association between licence status and medication errors. Arch Dis Child 2011;96(3):305-6. Epub 2010 Dec 3.

5. García-Bournissen F, Altcheh J, Giglio N, Mastrantonio G, Della Védova CO, Koren G. Pediatric clinical pharmacology studies in Chagas disease: focus on Argentina. Paediatr Drugs 2009; 11(1):33-7. Review.

6. AltchehJ,MoscatelliG, MoroniS, García-Bournissen F, Freilij $\mathrm{H}$. Adverse events after the use of benznidazole in infants and children with Chagas disease. Pediatrics 2011;127(1):e2128. Epub 2010 Dec 20.

7. www.clinical trials.gov. [Acceso mayo 2011]. 


\section{Cursos multimedia en línea} Multimedia courses

Entre las propuestas educativas formales ofrecidas en los últimos años han proliferado las de los cursos multimedia en línea y es esperable que este crecimiento continúe en forma exponencial.

Sin duda, estas experiencias resultan útiles entre las múltiples estrategias utilizadas para facilitar el desarrollo médico continuo. Sin embargo, teniendo en cuenta que la estrategia más eficaz es la enseñanza que se logra trabajando junto al paciente, resulta imprescindible analizar todo lo que el avance tecnológico hace posible al facilitar la comunicación, sus alcances y limitaciones. La información disponible sobrepasa frecuentemente las posibilidades de su utilización completa. No debemos caer en el facilismo de pensar que con más información lograremos necesariamente una mejor formación.

Frente a estas nuevas herramientas tecnológicas, urge considerar algunos aspectos aún no suficientemente evaluados en su plenitud, dado el relativo escaso lapso transcurrido desde su implementación.

Se hace necesario unificar criterios para que el docente, en el contexto de un modelo educativo en línea, comprenda cuál es su rol y responsabilidad en el aprendizaje de quienes toman el curso y en el éxito del programa educativo. Generalmente, estos modelos se sustentan en tres elementos: los materiales educativos, la asesoría o tutoría de un especialista en el tema y la evaluación como medio de certificación.

En primer lugar, se debe contar con una adecuada infraestructura administrativa, sistemas de gestión, fuentes de información y la responsabilidad de los organizadores para asegurar las condiciones logísticas, organizativas y tecnológicas necesarias para que el proceso educativo se desarrolle adecuadamente, sin descuidar los aspectos esenciales académicos y legales que den solidez, calidad y validez al programa.

Estos condicionamientos debieran ser integrados en pos de un objetivo: educar y lograr el mayor impacto posible en el cambio de conductas que se pretende instalar en la práctica médica cotidiana de quienes realizan el curso, sin olvidar que son ellos los protagonistas de su propia formación, con autonomía y autorregulación de acuerdo a los plazos previstos. El resultado debería ser la creación de comunidades de aprendizaje en línea, que posibiliten que los estudiantes se involucren con el curso y que el aprendizaje resulte activo, construido de forma social, y cuyos objetivos iniciales definidos por los docentes, en ocasiones, pueden ser superados.

Se debe resaltar la necesidad de complementar el componente teórico con actividades previamente planificadas para conseguir un aprendizaje más eficaz, significativo y de alto impacto sobre la competencia clínica. La utilización de herramientas como correo electrónico, foros de discusión y espacios de debate virtual fomentan un nuevo concepto de aprendizaje cooperativo, en el que se estimula el pensamiento crítico, y la aprehensión de nuevos conocimientos basados en la lectura, la reflexión y el intercambio de opiniones argumentadas.

Los participantes de un foro o debate pueden ingresar sus opiniones en cualquier momento dentro de un período determinado, generalmente amplio, y otros pueden responder, si lo consideran oportuno. Estos foros deben ser coordinados por un moderador, docente del curso.

En el caso del correo electrónico, su aplicación atenúa los inconvenientes de la saturación del tutor y le brinda más libertad y tiempo para preparar respuestas y ayudas más elaboradas, a la vez que permite atender en un plazo prudencial a todos los que realizan consultas.

En este nuevo escenario y con las facilidades de comunicación que presentan las nuevas tecnologías, el aislamiento potencial del que eran víctimas los alumnos que no podían acercarse a un centro de formación ha dejado de ser, ya que pueden utilizar espacios virtuales para interactuar con compañeros y tutores.

Debemos fomentar las posibilidades que brinda la tecnología y capacitar al docente de modo de combinar posibilidades didácticas y tecnológicas orientadas a potenciar el aprendizaje, recordando que los elementos tecnológicos constituyen un medio que facilita las tareas, pero que no debe condicionar la labor educativa que se pretende instalar. Lo importante son los conocimientos, las actitudes, las habilidades y destrezas que se pretende desarrollar a través del curso y la creatividad del docente para optimizar las potencialidades del medio para el logro de los objetivos previstos.

Existe la idea errónea de que la utilización de tecnologías para el aprendizaje implica me- 
nor carga de trabajo para el alumno. La realidad es que este trabajo a distancia es arduo y se basa en la lectura de contenidos, citas bibliográficas, libros de texto y guías didácticas impresas, participación en foros y desarrollo de actividades específicas. Los materiales educativos digitales, como libros electrónicos y aplicaciones multimedia, entre otros, se consideran elementos de apoyo útiles, pero no constituyen el núcleo medular en el proceso de formación a distancia en línea. Este tipo de materiales, cuyo ámbito de aplicación es el contexto de un curso o asignatura, deben enmarcarse para cubrir aquellos aspectos en los cuales las ventajas de su aplicación son prácticas y eficaces y deben ser cuidadosamente elegidos por los docentes como apoyo necesario para ampliar el conocimiento en la materia tratada. El aprovechamiento depende de la pertinencia y la dosificación dentro del contenido general del curso, una combinación que debe ser adecuada, pero principalmente práctica, para facilitar el logro de mejores resultados en la satisfacción y nivel de asimilación del estudiante.

Preparar un curso en línea, e implementarlo, requiere recursos humanos especialmente capacitados, que puedan interactuar con expertos informáticos, lo cual constituye una tarea nada fácil. No se trata solo de ofrecer uno o dos pdf.

En la educación a distancia en línea, la evaluación se toma de dos formas: la primera de ellas como estrategia de aprendizaje, la segunda como medio de certificación de conocimientos. En cualquier caso, la tecnología aplicada a la evaluación permite orientar el proceso de aprendizaje y potenciar las habilidades del estudiante. Son muy usuales las autoevaluaciones interactivas, en las que la retroalimentación argumentada e inmediata es importante y útil para que el estudiante pueda conocer el nivel alcanzado y, a la vez, corregir y conocer las partes del contenido en las que debe centrar más su atención. En las evalua- ciones en línea se suele disponer de un período determinado para responder un cuestionario con preguntas escogidas aleatoriamente y de carácter objetivo. A pesar del desarrollo tecnológico logrado, no se puede tener certeza de que la persona que realiza la evaluación en línea es quien debe ser; por esa razón, la evaluación en línea se aplica como estrategia de aprendizaje que lleva al alumno a poner en práctica lo aprendido y, a la vez, a saber cuáles son sus falencias, siendo útil en este contexto como evaluación formativa. La evaluación es un proceso permanente de información y reflexión sobre el proceso de producción de los aprendizajes.

En el momento actual, debería considerarse la necesidad de realizar encuentros presenciales integradores, para hacer una evaluación sumativa personalizada, objetivar el grado de cumplimiento de los objetivos establecidos en cada área y, por ende, el grado de desarrollo de la competencia que se busca alcanzar.

En cuanto a los organismos de ley que evalúan y califican estas actividades, les cabe la ardua tarea de unificar criterios respecto de los créditos que se otorgan a los cursos, la consistencia y el cumplimiento de los programas propuestos, las evaluaciones realizadas, etc. Existe una preocupación creciente por la calidad de estos cursos, y se deben elaborar estándares e instrumentos para evaluarlos. Sin duda, como Sociedad Científica tenemos un largo camino por recorrer en esta área tan importante como es la responsabilidad de debatir, en conjunto, los problemas que se relacionan con el desarrollo médico continuo, sin olvidar que, como entidad rectora, se está obligado a tener una opinión formada sobre el tema.

Dr. Luis Eduardo Urrutia

Hospital de Pediatría "Prof. Dr. Juan P. Garrahan" http:/ / dx.doi.org/10.5546/aap.2012.6 